PROCEEDINGS OF THE

AMERICAN MATHEMATICAL SOCIETY

Volume 134, Number 11, November 2006, Pages 3391-3393

S 0002-9939(06)08360-2

Article electronically published on June 6, 2006

\title{
A NOTE ON UNIFORMIZATION OF RIEMANN SURFACES BY RICCI FLOW
}

\author{
XIUXIONG CHEN, PENG LU, AND GANG TIAN
}

(Communicated by Richard A. Wentworth)

\begin{abstract}
We clarify that the Ricci flow can be used to give an independent proof of the uniformization theorem of Riemann surfaces.
\end{abstract}

In this note, we clarify that the Ricci flow can be used to give an independent proof of the uniformization theorem of Riemann surfaces. The key is the following simple observation stated in Lemma 0.1

When metric $g_{0}$ has positive curvature on $S^{2}$ or is a metric on a surface with genus bigger than 0, Hamilton proves that the volume-normalized Ricci flow $g(t)$ with $g(0)=g_{0}$ converges to a metric of constant curvature [Ha], however the proof in $\S 10$ of $\mathrm{Ha}$ of the fact that gradient shrinking soliton on $S^{2}$ has positive constant curvature, uses the uniformization theorem. There is another proof of the fact which uses the Kazdan-Warner identity (see p. 131 of [CK]), but the proof of the Kazdan-Warner identity uses the uniformization theorem. When $g_{0}$ does not have positive curvature on $S^{2}$, Chow proves that $g(t)$ will have positive curvature for large $t$ [Ch1]; in the proof the entropy formula is proved using the uniformization theorem. Later different proofs of the entropy estimate, which replaces the entropy formula, are given without using the uniformization theorem [Ch2, CW]. So the only time that the uniformization theorem is used for $g(t)$ to converge to a metric of constant curvature is when arguing that a gradient shrinking Ricci soliton on $S^{2}$ has positive constant curvature.

Lemma 0.1. Let $\left(\Sigma^{2}, g\right)$ be a two-dimensional complete Riemannian manifold with nontrivial Killing vector field $X$. Suppose $X$ vanishes at $O \in \Sigma$; then $\left(\Sigma^{2}, g\right)$ is rotationally symmetric.

Proof. Let $\Phi_{t}: \Sigma \rightarrow \Sigma, t \in(-\infty, \infty)$, be the isometry group generated by $X$, $\frac{d}{d t} \Phi_{t}(x)=X\left(\Phi_{t}(x)\right)$ and $\Phi_{0}(x)=x$. By assumption $\Phi_{t}(O)=O$ for all $t$. Hence the tangent of $\Phi_{t}$ induces an oriented linear isometry

$$
\left(\Phi_{t}\right)_{*}:\left(T_{O} \Sigma, g(O)\right) \rightarrow\left(T_{O} \Sigma, g(O)\right)
$$

Since the oriented linear isometry group of $\left(T_{O} \Sigma, g(O)\right)$ is $S^{1}$ and the map $t \rightarrow\left(\Phi_{t}\right)_{*}$ is a nontrivial homomorphism, there is a $t_{0}>0$ such that $\left(\Phi_{0}\right)_{*}=\left(\Phi_{t_{0}}\right)_{*}$. Note that isometry $\Phi_{t}$ is determined by $\left(\Phi_{t}\right)_{*}$ through minimal geodesic starting at $O$,

Received by the editors May 25, 2005 and, in revised form, June 3, 2005.

2000 Mathematics Subject Classification. Primary 53C44.

Key words and phrases. Ricci flow, uniformization of Riemann surfaces.

The authors were supported in part by NSF research grants.

(C)2006 American Mathematical Society 
so we have $\Phi_{t_{0}}=\Phi_{0}$. We have shown that there is a nontrivial $S^{1}$ isometric action on $(\Sigma, g)$. It is clear that if there is a ray starting at $O$, then $\Sigma$ is topologically $\mathbb{R}^{2}$. If there is not any ray starting at $O$, then $\Sigma$ is topologically $S^{2}$.

It was known that a complete gradient steady soliton in dimension two with positive curvature must be a cigar solution (see, for example, the editors' footnote on pp. 241-242 of [3CY] ). Now using the rotational symmetry from Lemma 0.1 , we can prove a similar proposition for gradient shrinking solitons without using the uniformization theorem.

Proposition 0.2. If $g$ is a gradient shrinking Ricci soliton on closed surface $\Sigma^{2}$, then $g$ has positive constant curvature.

Proof. Recall the gradient shrinking Ricci soliton equation $R_{i j}=c g_{i j}+\nabla_{i} \nabla_{j} f$ for some $c>0$ and some smooth function $f$ on $\Sigma$. We choose $c=1$ below. Since $\Sigma$ is closed, there is a point $O$ such that $\nabla f(O)=0$. Since $R_{i j}=\frac{1}{2} R g_{i j}$ in dimension 2 , it follows from the soliton equation that $\nabla f$ is a conformal vector field. Let $J$ be the almost complex structure on $T \Sigma$ defined by $90^{\circ}$ counterclockwise rotation. It is well known that $J(\nabla f)$ is a Killing vector field (see, for example, the editors' footnote on pp. 241-242 of [3CY] ). Hence by Lemma $0.1 \mathrm{~g}$ is rotationally symmetric: $g=d r^{2}+h(r)^{2} d \theta^{2}, 0 \leq r \leq A<\infty, 0 \leq \theta \leq 2 \pi$. From the proof of Lemma 0.1 we may assume $f=f(r)$. Using Gauss curvature $K_{g}=-\frac{h^{\prime \prime}}{h}$ the soliton equation becomes

$$
-\frac{h^{\prime \prime}}{h}=1+f^{\prime \prime} \quad-\frac{h^{\prime \prime}}{h}=1+\frac{h^{\prime} f^{\prime}}{h} .
$$

Integrating $f^{\prime \prime}=\frac{h^{\prime} f^{\prime}}{h}$ we get $f^{\prime}=a h$ for some constant $a$. Hence $-\frac{h^{\prime \prime}}{h}=1+a h^{\prime}$. Multiplying $h h^{\prime}$ and integrating over $[0, A]$, we get

$$
-\left.\frac{\left(h^{\prime}\right)^{2}}{2}\right|_{0} ^{A}=\left.\frac{h^{2}}{2}\right|_{0} ^{A}+a \int_{0}^{A} h\left(h^{\prime}\right)^{2} d r .
$$

Since the metric $d r^{2}+h(r)^{2} d \theta^{2}$ is smooth on $\Sigma$ at $r=0$ and $r=A$, it follows that $h(0)=h(A)=0$ and $h^{\prime}(0)=-h^{\prime}(A)=1$. So $a=0$ and $g$ is Einstein metric.

Hamilton proved that any possible nontrivial soliton on closed surface must be a gradient shrinking soliton (see the proof in $\S 10.1$ of [Ha] $)$. So there are no nontrivial solitons on closed surfaces.

In higher dimensions, one may have nontrivial shrinking Ricci solitons. However, the first and third authors can prove without using the uniformization theorem that any shrinking Kähler-Ricci soliton with positive bisectional curvature must be Kähler-Einstein [CT.

The second author thanks Bennett Chow for discussions about the editors' note in $3 \mathrm{CY}$.

\section{REFERENCES}

[3CY] H.D. Cao, B. Chow, S.C. Chu and S.T. Yau (editors), Collected papers on Ricci flow. Internat. Press, Somerville, MA (2003). MR2145154

[Ch1] B. Chow, The Ricci flow on the 2-sphere. J. Diff. Geom. 33 (1991), 325-334. MR.1094458 (92d:53036)

[Ch2] B. Chow, On the entropy estimate for the Ricci flow on compact 2-orbifolds. J. Diff. Geom. 33 (1991), 597-600. MR1094471 (92e:58228) 
[CK] B. Chow and D. Knopf, The Ricci flow: An introduction. AMS, Providence, RI (2004). MR2061425 (2005e:53101)

[CT] X.X. Chen and G. Tian, unpublished notes.

[CW] B. Chow and L.F. Wu, The Ricci flow on compact 2-orbifolds with curvature negative somewhere. Comm. Pure Appl. Math. 44 (1991), 275-286. MR1090433 (92g:53035)

[Ha] R. Hamilton, The Ricci flow on surfaces, Contemp. Math. 71 AMS, Providence, RI (1988), 237-262. MR0954419 (89i:53029)

Department of Mathematics, University of Wisconsin, Madison, Wisconsin 53706

E-mail address: xxchen@math.wisc.edu

Department of Mathematics, University of Oregon, Eugene, Oregon 97403

E-mail address: penglu@darkwing.uoregon.edu

Department of Mathematics, Princeton University, Princeton, New Jersey 08544

E-mail address: tian@math.princeton.edu 